\title{
Identification of novel diagnostic and prognostic miRNA signatures in endometrial cancer
}

\author{
Muralidharan Jayaraman ${ }^{1,2, *}$, Rangasudhagar Radhakrishnan ${ }^{1, *}$, Cara A. \\ Mathews ${ }^{1,3, *}$, Mingda Yan ${ }^{1}$, Sanam Husain ${ }^{4}$, Katherine M. Moxley ${ }^{1,3}$, Yong Sang \\ Song ${ }^{5}$, and Danny N. Dhanasekaran ${ }^{1,2}$ \\ ${ }^{1}$ Stephenson Cancer Center, The University of Oklahoma Health Sciences Center, Oklahoma City, OK, USA \\ 2 Department of Cell Biology, The University of Oklahoma Health Sciences Center, Oklahoma City, OK, USA \\ ${ }^{3}$ Department of Obstetrics and Gynecology, The University of Oklahoma Health Sciences Center, Oklahoma City, OK, USA \\ ${ }^{4}$ Department of Pathology, The University of Oklahoma Health Sciences Center, Oklahoma City, OK, USA \\ ${ }^{5}$ Department of Obstetrics and Gynecology, College of Medicine, Seoul National University, Seoul, S. Korea \\ *Equal Contribution
}

Correspondence to: Danny N. Dhanasekaran, email: danny-dhanasekaran@ouhsc.edu

Keywords: Endometrial cancer, miRNA, miR-142, miR-15a, therapy response

Received: June 01,2017 Accepted: June 21, 2017 Published: July 03, 2017

Copyright: Jayaraman et al. This is an open-access article distributed under the terms of the Creative Commons Attribution License (CC-BY), which permits unrestricted use, distribution, and reproduction in any medium, provided the original author and source are credited.

\section{ABSTRACT}

With the goal of identifying diagnostic and prognostic biomarkers in endometrial cancer, miRNA-profiling was carried out with formalin-fixed paraffin embedded (FFPE) tissue samples from 49 endometrial cancer patients. Results using an 84-cancer specific miRNA panel identified the upregulation of miR-141-3p and miR-96-5p along with a downregulation of miR-26, miR-126-3p, miR-23b, miR-195-5p, miR-374a and let-7 family of miRNAs in endometrial cancer. We validated the dysregulated expression of the identified miRNAs in a panel of endometrial cancer cell-lines. Immunohistochemical analysis of the tissue micro array derived from these patients established the functional correlation between the decreased expression of tumor suppressive miRNAs and their target oncogenes: ERBB2, EGFR, EPHA2, BAX, GNA12, GNA13, and JUN. Comparative analysis of the samples from the patients with extended progression-free survival (PFS) ( $>21$ months) versus the patients with the PFS of $<21$ months indicated increased expression of tumor suppressive miR-142-3p, miR-142-5p, and miR-15a-5p in samples from extended PFS patients. In addition to defining a specific set of miRNAs and their target genes as potential diagnostic biomarkers, our studies have identified tumor suppressive miR-142 cluster and miR$15 \mathrm{a}$ as predictors of favorable prognosis for therapy response in endometrial cancer.

\section{INTRODUCTION}

Endometrial cancer is one of the major gynecological cancer that will be affecting more than 61,000 patients with the anticipated death of 10,920 patients in 2017 [1]. While early detection has significantly increased the overall survival rate, $20 \%$ of the cases have poor prognosis with a median survival rate of just an year [2]. This is primarily due to the resistance to chemotherapy and disease recurrence. No precise molecular tags are available at this time to be used as a good diagnostic or prognostic biomarker for therapy resistance and disease recurrence in endometrial cancer [3-6]. Identifying a prognostic marker will greatly benefit the patients since it can be used as a predictor for therapy response so as to target treatment to the patients who would be greatly benefited from the specific adjuvant therapy. With the goal of identifying such a prognostic marker, we focused on defining the changes in miRNA profiles that could be associated with therapy resistance in endometrial cancer patients. In this study, we investigated the changes in miRNA profiles using FFPE-samples derived from a cohort of endometrial cancer patients. Using FFPE-samples from patients who had progressive disease during or shortly following 
chemotherapy and patients who remained without disease recurrence, expression profiles of miRNAs were analyzed using a cancer-specific 84-miRNA analysis panel.

Our results presented here indicates that the endometrial cancer tissues showed an increase in the expression of several oncomiRs with a concomitant decrease in the expression of tumor suppressor miRNAs compared to control samples. Overexpression of known oncomiRs include miR-32-5p, miR-96-5p, miR-141$3 p$, miR-142-5p, and miR-210. Whereas, the tumor suppressive miRNAs that showed decreased expression in endometrial cancer tissue are: miR-26, miR-126-3p, miR-23b, miR-195-5p, miR-374a and let-7 family of miRNAs. Validation of the expression of these miRNAs in endometrial cancer cell lines HEC1A, HEC1B RL95, Ishikawa and/or HEC50 indicated a similar increased expression of the representative oncomiRs along with the downregulation of tumor suppressive miRNAs, thus validating them with the oncogenic signature of endometrial cancer. Our results also indicate that the expression of the tumor-specific miRNAs can be correlated with their effect on their respective target mRNAs indicated by an increase in the expression of several oncogenic proteins such ERBB2, EGFR, EPHA2, BAX, GNA12, GNA13, and JUN, further substantiating the validity of these miRNAs as potential tumor-specific biomarkers in endometrial cancers. Differential analysis of miRNA profile between recurrent and non-recurrent patients indicated that the upregulation of the tumor suppressor miR-142-3p, miR-142-5p, and miR-15a-5p along with a drastically increased expression of miR-96$5 p$ was observed in the samples derived from patients who showed a PFS of more than 21 months. In contrast, the samples derived from patients with PFS of less than 21 months showed little or no expression of miR-142$3 p$, miR-142-5p, and miR-15a-5p and relatively weaker expression of miR-96-5p. Thus, our studies presented here identifies the potential role of miR-142-3p, miR-142-5p, and miR-15a-5p, as favorable prognostic indicators for therapy response in endometrial cancer.

\section{RESULTS AND DISCUSSION}

\section{Expression profiling of miRNAs in endometrial cancer samples}

To identify a panel of miRNAs that can serve as a diagnostic and/or prognostic marker in endometrial cancer, we carried out a retrospective cohort analysis. Expression of miRNA in endometrial cancer patients using archived FFPE samples from 49 patients who underwent surgery for advanced endometrial cancer was carried out. Based on the survival and recurrence data, the subjects were divide into two clinical groups namely, "responders to chemotherapy" and "non-responders to chemotherapy". The responder group $(n=26)$ is defined by the subjects without disease recurrence for more than 21 months whereas the nonresponders $(n=23)$ represented the subjects whose disease continued to progress either during chemotherapy $(n=6)$ or soon after the completion of chemotherapy $(n=17)$. While the responders had progression free survival with a median value of 52 months, all of the non-responders succumbed to the disease by $3-21$ months. Based on the quality of the FFPE blocks, specimens from 16 responders and 20 non-responders were processed for miRNA profile analysis. Normal endometrial tissue from six FFPE specimens were used as the control group. The miRNA profiling was carried out by PCR methods to monitor the expression of 84 well-characterized cancer related miRNAs. As shown in Fig. 1, the samples from cancer tissues showed altered expression profile of several specific miRNAs (Figure 1A). A comparative scatter blot between control and patient tumor samples, derived from this dataset identified the unique set of miRNAs that showed altered expression in endometrial cancer (Figure 1B). Our results indicated that only miR-141-3p and miR-96-5p showed significant increase in cancer tissue samples compared to normal tissue sample by two folds. Increased expression seen with miR-96-5p, miR-142-5p, and miR-32-5p were not statistically significant. It is of interest to note here that miR-141-3p [8] and miR-96$5 \mathrm{p}$ have been identified as OncomiRs in many cancers [8-21]. In striking contrast, endometrial cancer tissue samples showed a significant decrease in fourteen tumorsuppressive miRNAs (Figure 1C). It is of interest to note here that all of the fourteen downregulated miRNAs, namely, miR-26a-5p [22, 23], miR-150-5p [24, 25], let7f-5p [26, 27], miR-26b-5p [22, 23, 28], let-7c-5p [26, 27], miR-23b-5p [29-31], miR-126-3p [32], miR-125b-5p [33], hsa-miR-195-5p [34, 35], miR-424-5p [36, 37], hsamiR-374a-5p [38-40], let-7a-5p [26, 27] , let-7e-5p [26, 27], and miR-125a-5p [41, 42] are known to have tumorsuppressive function in many cancers. Taken together with the upregulated genes, our results identify a panel of miRNAs that correlates with the tumor phenotype. Our results showing the upregulation of miR-141 miR-96, and miR-32 in endometrial cancer are in agreement with a previous report that identified the increased expression of these miRNAs in endometrial cancer [43]. Our studies presented here extend this further with a novel finding that identify an array of fourteen tumor-suppressive miRs that are downregulated in endometrial cancer.

\section{Validation of differentially expressed miRNAs}

In view of the critical oncogenic responses regulated by these miRNAs (Table 1), we sought to validate the array results with the use of endometrial cancer cell lines. This was carried out using a panel of endometrial cancer cell 
lines consisting of HEC1A, HEC1B, HEC50, Ishikawa, or RL95 cell lines along with fallopian tube derived epithelial cell line, FTE-188, as control. Expression of the candidate miRNAs in these cell lines were monitored by RT-qPCR analysis. As shown in Fig. 2A, the miR-32-5p, miR-96-5p, miR-141-3p, and miR-210 showed increased expression in endometrial cancer cell lines compared to the control cells FTE188 cells. Whereas, the miRNAs with tumor-

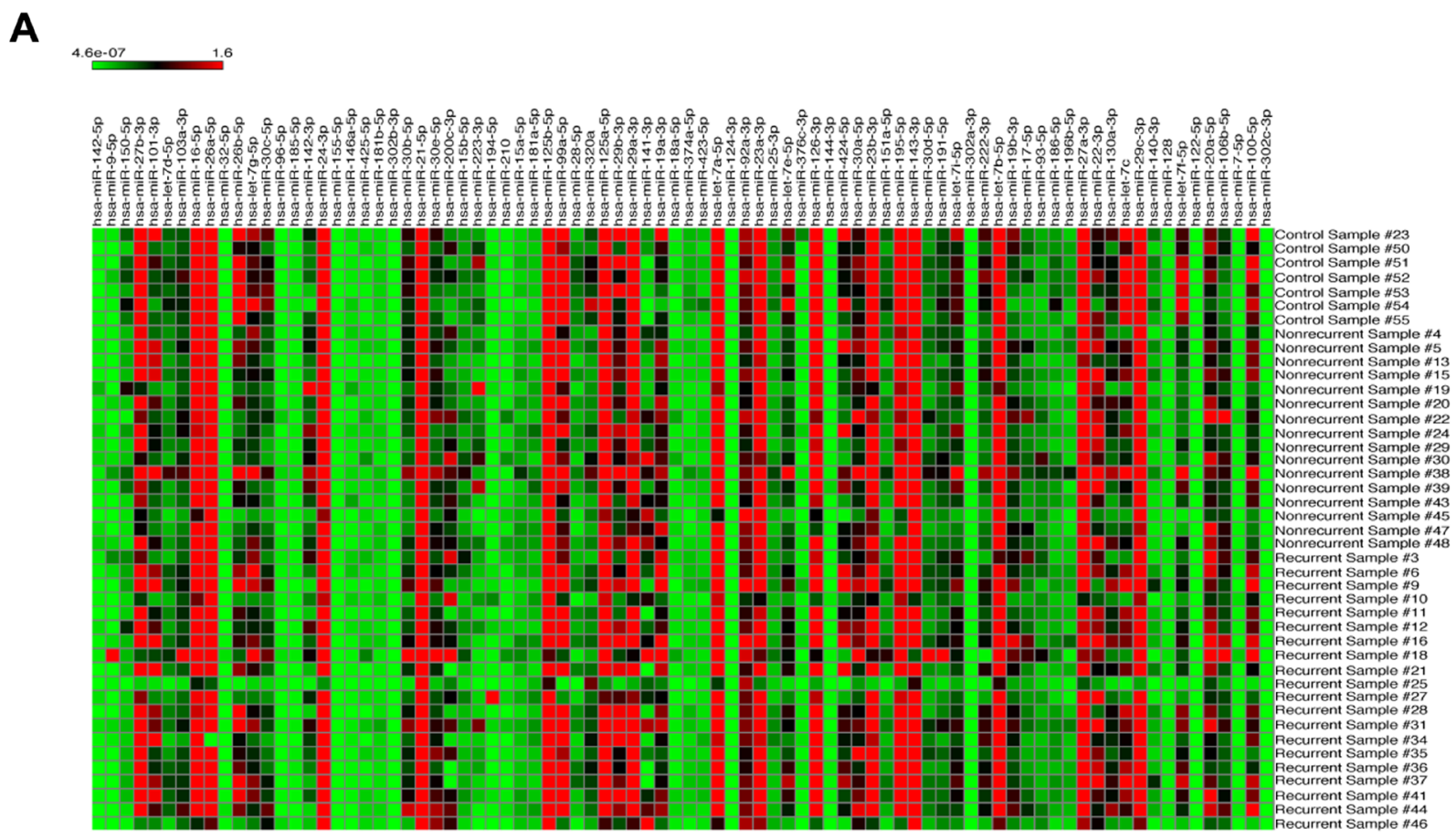

B

Tumor Vs Control

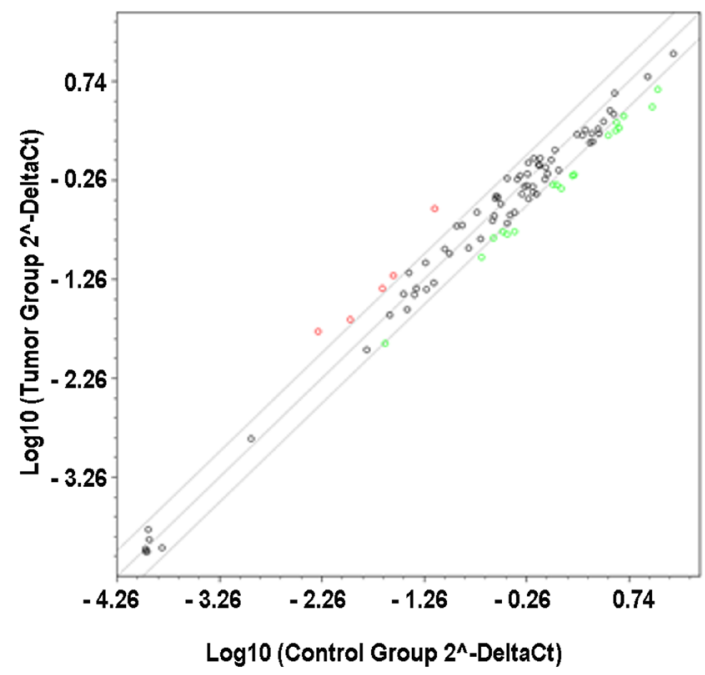

C

\section{Upregulated miRNAs}

\begin{tabular}{|l|l|}
\hline miRNA ID & Fold Regulation \\
\hline hsa-miR-141-3p & $4.1^{*}$ \\
\hline hsa-miR-96-5p & $3.2^{\star}$ \\
\hline hsa-miR-210-3p & 2.1 \\
\hline hsa-miR-142-5p & 2.1 \\
\hline hsa-let-32-5p & 2.1 \\
\hline
\end{tabular}

\section{Downregulated miRNAs}

\begin{tabular}{|l|l|}
\hline miRNA ID & Fold Regulation \\
\hline hsa-miR-26a-5p & $-3.1^{\star \star}$ \\
\hline hsa-miR-150-5p & $-2.7^{\star \star}$ \\
\hline hsa-let-7f-5p & $-2.6^{\star \star \star}$ \\
\hline hsa-miR-26b-5p & $-2.6^{\star \star \star}$ \\
\hline hsa-let-7c-5p & $-2.5^{\star \star}$ \\
\hline hsa-miR-23b-3p & $-2.4^{\star \star}$ \\
\hline hsa-miR-126-3p & $-2.3^{\star \star}$ \\
\hline hsa-miR-125b-5p & $-2.3^{\star \star}$ \\
\hline hsa-miR-195-5p & $-2.2^{\star \star \star}$ \\
\hline hsa-miR-424-5p & $-2.2^{\star}$ \\
\hline hsa-miR-374a-5p & $-2.1^{\star \star *}$ \\
\hline hsa-let-7a-5p & $-2.0^{\star \star}$ \\
\hline hsa-let-7e-5p & $-2.0^{\star \star}$ \\
\hline hsa-miR-125a-5p & $-2.0^{\star \star}$ \\
\hline
\end{tabular}

Figure 1: Expression analysis of miRNAs in FFPE samples from endometrial cancer patients. Heat map and scatter plot images represent results from miRNA profiling using FFPE samples derived from normal or endometrial cancer patients by RT-qPCR. A. Heat map shows differential miRNA expression profiles between normal and endometrial cancer patient derived samples. Upregulated genes are shown in red and downregulated genes are in green. B. In the scatter plot, upregulated miRNAs ( $>2$-fold) are denoted by red circles and downregulated miRNAs $(<2$-fold) are denoted by green open circles. $C$. Upregulated and downregulated miRNAs in FFPE samples from endometrial cancer tissue along with the fold changes are listed. Significant differences in the fold changes are indicated by p-values: $*_{-}<0.05 ; * *-<0.01 ; * * *-<0.001$. 
suppressive effect, namely, miR-23b-3p, miR-26a-5p, miR-26b-5p, let-7f-5p, let-7c-5p, miR125a-5p, miR-374a and miR-195-5p showed a decreased expression (Figure 2B). The expression profiles of the putative oncomiRs as well as the tumor suppressor miRs were quite similar to their expression profile in endometrial cancer tissue thereby validating the results obtained using the FFPE samples from the endometrial cancer patients.

\section{Expression Analysis of miRNA-target proteins}

MiRNAs regulate gene expression through RISCmediated mRNA degradation as well as translational repression [44, 45]. Therefore, we sought to verify the functional relevance of the altered miRNA expressions especially that of tumor-suppressive miRs - in endometrial cancer pathobiology by monitoring the expression of the target genes in cancer tissue sample. To test whether the decreased expressions of the tumor-suppressive miRs correlate with the increased expression of growth promoting target genes, a tissue micro array (TMA)based immunohistochemical analysis was carried out. Using the list of downregulated miRNAs identified here, TarBase v7.0 database (http://www.diana.pcbi.upenn.edu/ tarbase) was searched for experimentally identified target proteins. A TMA, constructed from the FFPE samples used in this study, was probed with antibodies to specific target gene products. Using this TMA, the expression of ERBB2, EGFR, EPHA2, GNA12, GNA13, JUN, and SET messages targeted by miR-26a-5p (EPHA2), miR-26b5p (EPHA2, GNA13), let-7f-5p (GNA13), miR-23b-3p (EPHA2, GNA12), miR-126-3p (GNA13), miR-374-5p (JUN), and miR-125a-5p (Jun, ERBB2, SET, EGFR), were

A
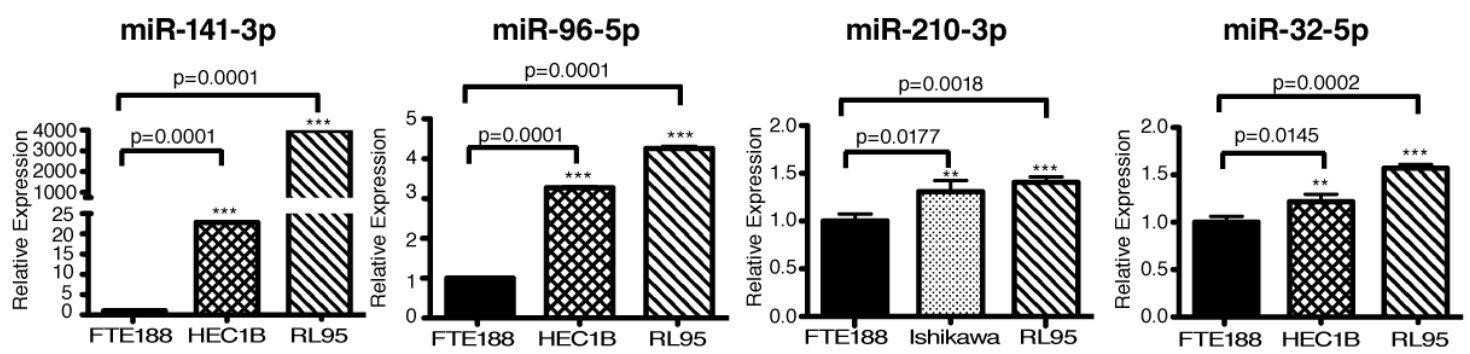

B
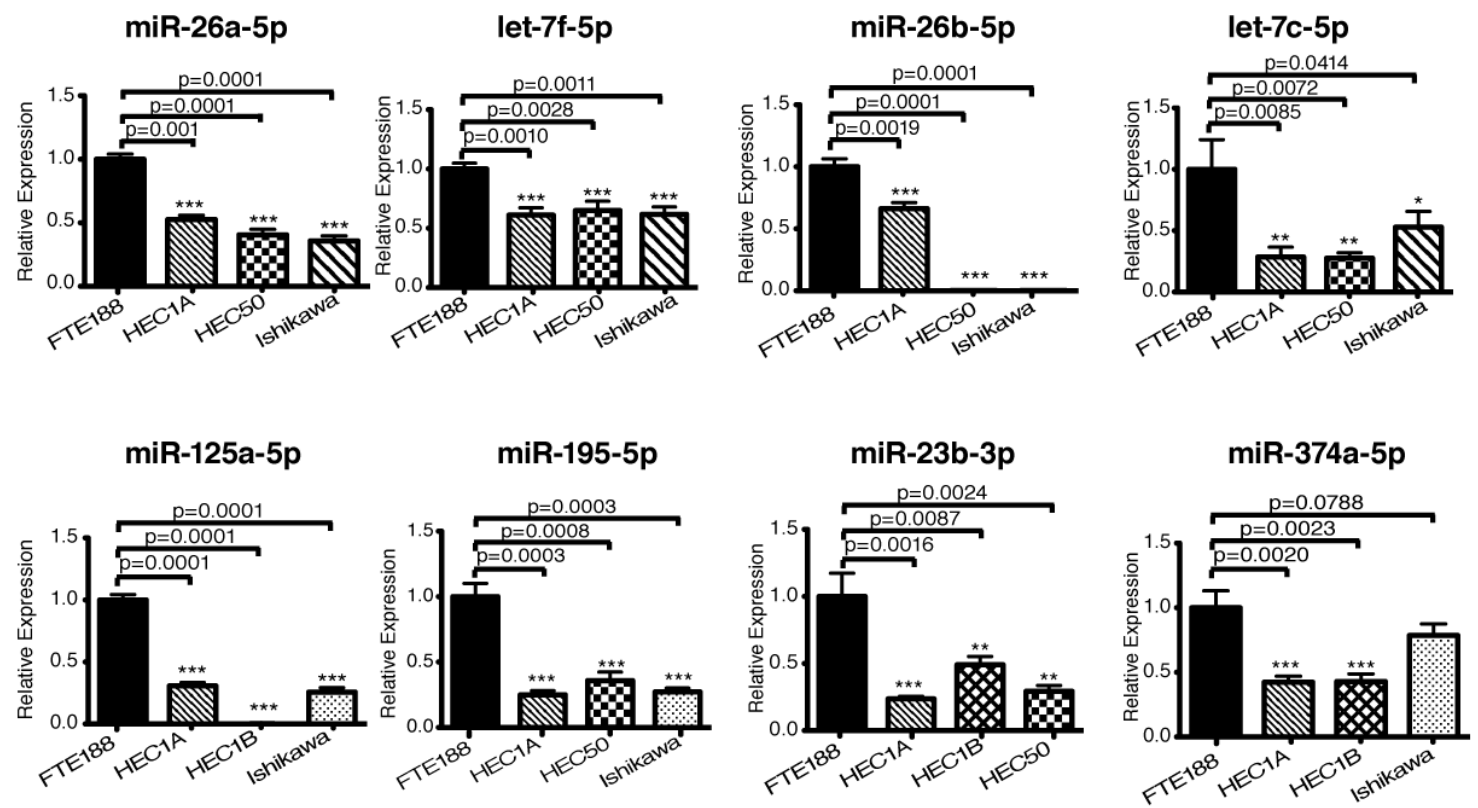

Figure 2: Expression analysis of miRNAs in endometrial cancer cell lines. A. Expression of upregulated miRNAs (miR-32-5p, miR-96-5p, miR-141-3p, miR-142-5p, and miR-210) in HEC1B, RL95 and Ishikawa cells in comparison with the normal fallopian tube derived epithelial cells (FTE188) are presented. Values are presented as Mean \pm SEM ( $n=3)$. B. Expression of downregulated miR-26a-5p, let-7f-5p, miR-26b-5p, let-7c-5p, miR-125a-5p, miR-195-5p, miR-23b-3p and miR-374a-5p were determined by RT-qPCR in HEC1A, HEC1B, HEC50 and Ishikawa cells in comparison with the FTE188 control cells are presented (Mean \pm SEM; $n=3$ ). 
monitored. Our results indicated the increased expression of ERBB2, EGFR, EPHA2, GNA12, GNA13, JUN, and SET proteins in cancer tissue samples to endometrial cancer patients compared to normal endometrial tissue (Figure 3). This correlated well with the decreased expressions of the miRNAs that could target the mRNAs that encode these proteins. Although the expression of a few of these proteins such as EGFR, EphA2, ERBB2, and c-Jun have been correlated previously with poor prognosis in endometrial cancer [46-49], the mechanism involved in the upregulation of these oncogenic proteins have not been fully understood. In this context, our observation that the miRNAs that regulate the levels and the activity of mRNAs, and subsequently the levels of the proteins that are encoded by them is highly significant in that it provides the mechanism by which these genes are regulated. Although the specific roles of GNA12, GNA13, and $S E T$ genes in endometrial cancer are not known at present, these genes have been identified as oncogenes that do promote tumorigenesis, tumor progression, and metastasis in many different cancers. Thus, our results identify for the first time a potential role for the tumor suppressor miRs and their targeted effect on specific oncogenic proteins in endometrial cancer.

\section{Therapy response specific miRNA signature}

In an attempt to infer more information about these miRNAs either as diagnostic or prognostic markers, miRNA profiles were compared between the patients who were stratified as therapy responders (PFS $>21$ months) and non-responders (PFS $<21$ months). Canceling out the miRNAs that were common to both groups indicated, there was no significant difference between the two groups in the profile of downregulated miRNAs
(Figure 4). Although miR-150-5p, miR-223-3p, and miR424-5p appeared specifically downregulated in "nonresponder" group, further analysis of the data indicated the samples from the "responder" group also showed a similar (albeit reduced fold decrease, $<2$-fold and $>1.5$ fold) downregulation (Figure 4). Likewise, the "unique" downregulated miRNA signatures seen with miR-125a$5 p$ and miR-30c-5p in the "responder" group was due to the 2-fold cutoff imposed on the analysis of the data. In fact, these miRNAs showed 1.8-fold and 1.6-fold decrease in the samples from the "non-responder" group. Thus, there does not seem to be a significant difference in the downregulated miRNAs between both the groups of patients. In contrast, the comparison of upregulated miRNAs indicated that the samples from patients with better prognosis showed the upregulation of miRNA, miR-142-3p, miR-142-5p, and miR-15a-5p (Fig. 4). In contrast, the samples derived from patients with PFS of less than 21 months showed little or no expression of miR$142-3 p$, miR-142-5p, and miR-15a-5p. It is worth noting here that although both the groups showed an increase in the expression of miR-96-5p, the "responder group" showed a relatively stronger expression profile (6-fold increase versus 1.9-fold increase). Considering the role of miR-96 in inhibiting cell proliferation [50] and enhancing chemsensitivity to DNA-damaging therapeutic such as cisplatin [51], it is possible that the increased expression of miR-96-5p along with the miR-142 cluster and miR-15a predicts good prognosis in endometrial cancer.

In light of the known tumor suppressor and chemosensitization roles of miR-142-3p, miR-142-5p, and miR$15 \mathrm{a}-5 \mathrm{p}$, our results point to their potential role in therapy response and identifies them as predictive biomarkers for therapy response. It has been shown that miR-142$3 p$ acts as a tumor suppressor miR that inhibits tumor progression in breast cancer as well as non-small cell lung

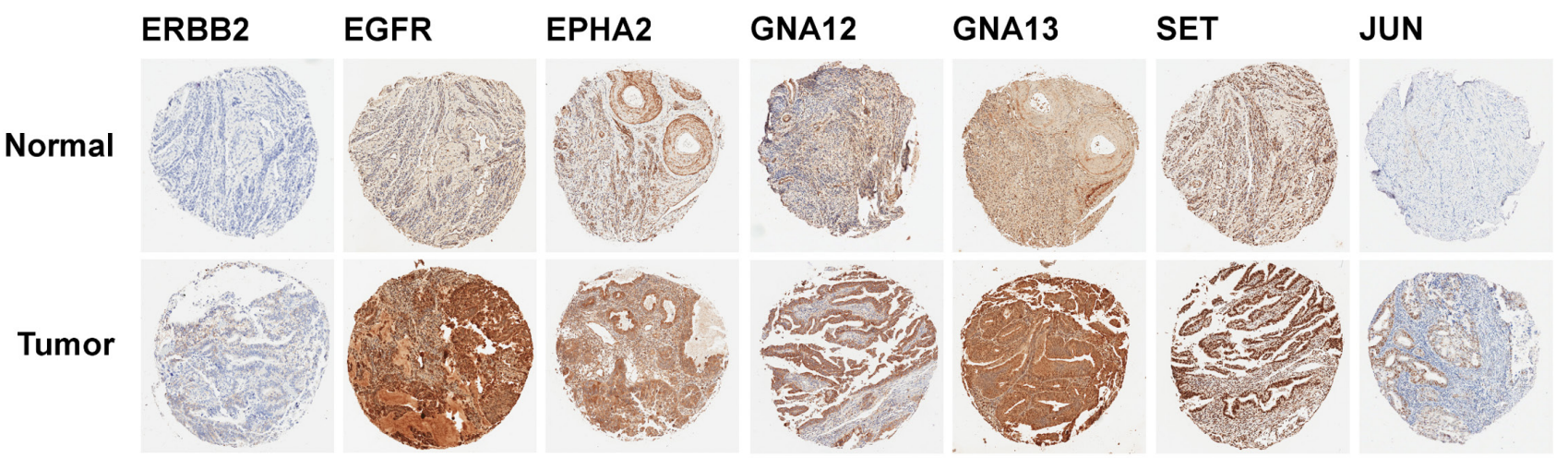

Figure 3: IHC analysis for the expression of target proteins in the endometrial cancer TMA. A TMA, constructed from the patient FPPE blocks of $1 \mathrm{~mm}$ diameter cores was subjected to IHC analysis using antibodies to specific proteins encoded by the representative target genes for the putative tumor suppressor miRs as detailed in the text. Sample micrographs of the TMA spots developed with 3, 3'-diaminobenzidine (DAB)/HRP staining (brown) for the respective protein are presented; magnification: X 10). 
carcinoma [52, 53]. Likewise, it has been observed that miR-142-5p inhibits cell growth and induces apoptosis in many different cancer cell lines $[18,54,55]$. Tumor

\section{A}

\section{Responders Vs Control}

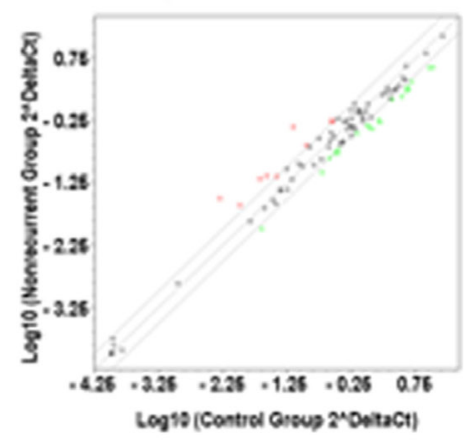

Upregulated miRNAs

\begin{tabular}{|l|l|}
\hline miRNA I0 & Fold Rogulation \\
\hline hsa-miR-96-5p & $6.2^{*}$ \\
\hline hsa-miR-141-3p & $\mathbf{5 . 7 ^ { * }}$ \\
\hline hsa-miR-142-5p & $2.9^{*}$ \\
\hline hsa-miR-210 & 2.5 \\
\hline hsa-miR-142-3p & 2.0 \\
\hline hsa-miR-32-5p & $2.0^{*}$ \\
\hline hsa-miR-15a-5p & $1.9^{*}$ \\
\hline
\end{tabular}

Downregulated miRNAs

\begin{tabular}{|l|l|}
\hline miRNA ID & Fold Regulation \\
\hline hsa-miR-26b-5p & $-2.8^{* *}$ \\
\hline hsa-let-7f-5p & $-2.8^{* *}$ \\
\hline hsa-let-7c & $-2.7^{*}$ \\
\hline hsa-miR-23b-3p & $-2.4^{*}$ \\
\hline hsa-miR-125b-5p & $-2.3^{* *}$ \\
\hline hsa-miR-195-5p & $-2.3^{* * *}$ \\
\hline hsa-miR-374a-5p & $-2.3^{* *}$ \\
\hline hsa-miR-26a-5p & $-2.2^{* *}$ \\
\hline hsa-miR-126-3p & $-2.2^{* *}$ \\
\hline hsa-let-7e-5p & $-2.1^{* *}$ \\
\hline hsa-miR-125a-5p & $-2.1^{* *}$ \\
\hline hsa-miR-30c-5p & $-2.0^{* *}$ \\
\hline
\end{tabular}

suppressor role of miR-142-3p and miR-142-5p has been further validated by the observation that the expression of both miR-142-3p and miR-142-5p are down regulated in

\section{B}

\section{Non-responders Vs Control}

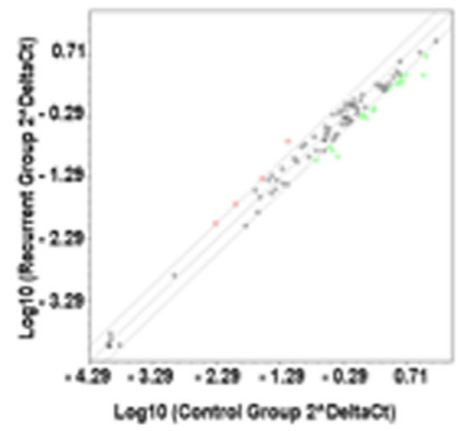

\section{Upregulated miRNAs}

\begin{tabular}{|l|l|}
\hline miRNA ID & Fold Rogulation \\
\hline hsa-miR-141-3p & 2.9 \\
\hline hsa-miR-96-5p & 1.9 \\
\hline hsa-miR-32-5p & 1.8 \\
\hline hsa-miR-210 & 1.8 \\
\hline
\end{tabular}

\section{Downregulated miRNAs}

\begin{tabular}{|l|l|}
\hline miRNA ID & Fold Regulation \\
\hline hsa-miR-26a-5p & $-3.8^{* *}$ \\
\hline hsa-miR-223-3p & $-3.7^{* *}$ \\
\hline hsa-let-7f-5p & $-2.5^{* * *}$ \\
\hline hsa-miR-150-5p & $-2.5^{* *}$ \\
\hline hsa-let-7c & $-2.4^{*}$ \\
\hline hsa-miR-23b-3p & $-2.4^{*}$ \\
\hline hsa-miR-26b-5p & $-2.3^{*}$ \\
\hline hsa-miR-126-3p & $-2.3^{* *}$ \\
\hline hsa-let-7a-5p & $-2.0^{* *}$ \\
\hline hsa-miR-195-5p & $-2.0^{* *}$ \\
\hline hsa-miR-424-5p & -2.0 \\
\hline hsa-miR-374a-5p & $-2.0^{* * *}$ \\
\hline
\end{tabular}

Figure 4: Expression analysis of miRNAs in FFPE samples from different therapy response groups. MiRNAs expression profiles in FFPE samples derived from endometrial cancer patients grouped as "non-recurrent/responder" (PFS > 21 months) and recurrent/ non-responder (PFS $<21$ months) were carried out using Human miFinder miScript miRNA PCR array kit by RT-qPCR. Red open circles indicate each miRNA expression, upregulated miRNA $(>2$-fold) as red and downregulated miRNA ( $<-1.8$-fold) as green open circles. A. Scatterplot (Upper Panel) represents results from miRNA profiles showing differentially expressed miRNAs in the endometrial cancer tissue derived from the "responder" group compared to normal tissue. List of miRNAs and the fold changes are presented in the lower panels. B. Scatter plot in the upper panel shows the differential expression of miRNAs between the endometrial cancer tissue from recurrent/non-responder group and normal tissue. Lower panels list the differentially expressed miRNAs and the associated fold changes. Statistically significant difference in the fold changes are indicated by p-values: $*_{-}<0.05 ; * *-<0.01 ; * * *-<0.001$. 
hepatocellular carcinoma [56]. Similarly, miR-15a-5p has been shown to act as a tumor suppressor through its ability to silence the expression of many growth promoting oncogenes including C-Jun [57-59]. In fact, it has been shown that the upregulation of miR-142 improves drug sensitivity in several cancers including acute myelogenous leukemia and non-small cell lung cancer [60, 61]. MiR15a has also shown to enhance the chemosensitivity of cancer cells to therapeutic agents including cisplatin $[62,63]$. Although, such a role for miR-142 and miR$15 \mathrm{a}$ has not been established in endometrial cancer, it is possible that the upregulation of these miRNAs enhances the chemosensitivity and contributes to the increased PFS time seen in the "responder" category of patients. Cumulatively, these observations along with our results point to the potential utility of these miRNAs as miRNA replacement therapy for endometrial cancer. In addition to defining a specific set of miRNAs and their target genes that can potentially serve as diagnostic biomarker for endometrial cancer, our studies have identified the tumorsuppressive miR-142 cluster and miR-15a as predictive biomarkers for good prognosis. Further studies should define the target genes and mechanism(s) by which these miRNAs confer clinical advantage to endometrial cancer patients.

\section{MATERIALS AND METHODS}

\section{Cell lines}

Endometrial cancer cell line, HEC1A, (ATCC, Manassa, VA) was cultured in McCoy's 5a medium supplemented with 10\% FBS (Norcross, GA), 50 U/ $\mathrm{mL}$ penicillin, $50 \mu \mathrm{g} / \mathrm{mL}$ streptomycin (Mediatech, Manassas, VA) at $37^{\circ} \mathrm{C}$ in a $5 \% \mathrm{CO}_{2}$ incubator. Similarly, HEC1B (ATCC, Manassa, VA) was cultured in Eagle's Minimum Essential Medium supplemented with $10 \%$ FBS, and penicillin-streptomycin. HEC50 and Ishikawa (a kind gift from Dr. Kimberly K. Leslie, The University of Iowa) was obtained and cultured in DMEM with the indicated supplements as above. The control cell line, FTE188 was cultured in a mixed media at a ratio of 50:50::MCDB105:M199, supplemented with 10\% FBS, Epidermal growth factor $(10 \mathrm{ng} / \mathrm{ml}$; Sigma, St. Louis, $\mathrm{MO}$ ), and $50 \mathrm{U} / \mathrm{mL}$ penicillin, $50 \mu \mathrm{g} / \mathrm{mL}$ streptomycin.

\section{RNA extraction and miRNA Analysis}

Total RNA including miRNA was extracted from 4x5 micron FFPE sections using Qiagen RNeasy FFPE kit (Qiagen, Valencia, CA) by following the manufacturer's instructions. cDNA synthesis was carried out using a
miScript II RT cDNA synthesis kit (Qiagen, Valencia, $\mathrm{CA}$ ) and the quality of miRNA and efficiency of cDNA synthesis was checked using a miRNA-QC PCR array (Catalog \# MIHS-999Z; Qiagen, Valencia, CA). Real-time quantitative PCR (RT-qPCR) was carried out using the cDNA from the above step using miScript SYBR green PCR kit (Bio-Rad, Carlsbad, CA) in a Bio-Rad CFX96 Real time PCR Detection System. The raw Cq values from this assay were exported and data analyzed using software available at http://pcrdataanalysis.sabiosciences.com/ mirna following the manufacturer's instructions. Only the samples that passed quality control (QC) PCR array were used in miScript Human miFinder miScript miRNA PCR array kit (MIHS-001Z; Qiagen, Valencia, CA) that profiles the expression of 84 different miRNAs as listed by the manufacturer (https://www.qiagen.com/ch/shop/ pcr/primer-sets/miscript-mirna-pcr-arrays/?catno=MIHS001Z\#geneglobe). They are:

hsa-let-7a-5p, hsa-let-7b-5p, hsa-let-7c-5p, hsalet-7d-5p, hsa-let-7e-5p, hsa-let-7f-5p, hsa-let-7g-5p, hsa-let-7i-5p, hsa-miR-100-5p, hsa-miR-101-3p, hsamiR-103a-3p, hsa-miR-106b-5p, hsa-miR-122-5p, hsa-miR-124-3p, hsa-miR-125a-5p, hsa-miR-125b-5p, hsa-miR-126-3p, hsa-miR-128-3p, hsa-miR-130a-3p, hsa-miR-140-3p, hsa-miR-141-3p, hsa-miR-142-5p, hsa-miR-142-3p, hsa-miR-143-3p, hsa-miR-144-3p, hsa-miR-146a-5p, hsa-miR-150-5p, hsa-miR-151a-5p, hsa-miR-155-5p, hsa-miR-15a-5p, hsa-miR-15b-5p, hsamiR-16-5p, hsa-miR-17-5p, hsa-miR-181a-5p, hsa-miR181b-5p, hsa-miR-185-5p, hsa-miR-186-5p, hsa-miR-18a5p, hsa-miR-191-5p, hsa-miR-194-5p, hsa-miR-195-5p, hsa-miR-196b-5p, hsa-miR-19a-3p, hsa-miR-19b-3p, hsamiR-200c-3p, hsa-miR-20a-5p, hsa-miR-21-5p, hsa-miR210-3p, hsa-miR-22-3p, hsa-miR-222-3p, hsa-miR-2233p, hsa-miR-23a-3p, hsa-miR-23b-3p, hsa-miR-24-3p, hsa-miR-25-3p, hsa-miR-26a-5p, hsa-miR-26b-5p, hsamiR-27a-3p, hsa-miR-27b-3p, hsa-miR-28-5p, hsa-miR29a-3p, hsa-miR-29b-3p, hsa-miR-29c-3p, hsa-miR-302a3p, hsa-miR-302b-3p, hsa-miR-302c-3p, hsa-miR-30a-5p, hsa-miR-30b-5p, hsa-miR-30c-5p, hsa-miR-30d-5p, hsamiR-30e-5p, hsa-miR-32-5p, hsa-miR-320a, hsa-miR374a-5p, hsa-miR-376c-3p, hsa-miR-423-5p, hsa-miR424-5p, hsa-miR-425-5p, hsa-miR-7-5p, hsa-miR-9-5p, hsa-miR-92a-3p, hsa-miR-93-5p, hsa-miR-96-5p, hsamiR-99a-5p

The QC-passed cDNA samples were subjected to RT-qPCR using the miScript SYBR green PCR kit (Bio-Rad, Carlsbad, CA) in a Bio-Rad CFX96 Real time PCR system. The raw $\mathrm{Cq}$ values from these assays were exported and data analyzed using software available at http://pcrdataanalysis.sabiosciences.com/mirna following the instructions. The data was normalized to the house keeping genes in the array and miRNAs that were up- or down-regulated by 1.8-fold compared to the control group along with the p-values were extracted from the software. 


\section{Expression of miRNA in cell lines}

Endometrial cancer cell lines HEC1A, HEC1B, HEC50, Ishikawa and FTE188 control cells were cultured and collected during their log phase of growth and total RNA including the miRNA was extracted using RNAeasy plus mini kit (Qiagen, Valencia, CA). The RNA was quantitated using a nanodrop and cDNA synthesis was carried out as described above. RT-qPCR analysis was carried out using the miScript Primer Assays (Qiagen, Valencia, CA) specific for the miRNA of interest. The data were analyzed in CFX Manager software and exported to GraphPad Prism (La Jolla, CA) where graphs and statistical analyses were done.

\section{Immunohistochemical staining of tissue microarray (TMA)}

TMA was constructed at the Stephenson Cancer Center's tissue pathology core using Veridiam VTA-100 Tissue Arrayer [7]. IHC-staining of the TMA with the antibodies of interest was carried out at the SCC tissue pathology core that utilizes automated Leica Bond III for IHC staining. In brief, FFPE tissues were sectioned at desired thickness $(4 \mu \mathrm{m})$ and mounted on positively charged slides. The slides were dried overnight at room temperature and incubated at $60^{\circ} \mathrm{C}$ for 45 minutes followed by deparaffinization and rehydration in an automated Multistainer (Leica ST5020). Subsequently, these slides were transferred to the Leica Bond-III ${ }^{\mathrm{TM}}$, treated for target retrieval at $100^{\circ} \mathrm{C}$ for 20 minutes in a retrieval solution, either at $\mathrm{pH} 6.0$ or $\mathrm{pH} 9.0$. Endogenous peroxidase was blocked using peroxidase-blocking reagent, followed by the selected primary antibody incubation for 60 minutes. For the secondary antibody, post-primary IgG-linker and/or Poly-HRP IgG reagents was used. 3, 3'-diaminobenzidine tetrahydrochloride (DAB) was used as the chromogen and the slides were counterstained with hematoxylin. Completed slides were dehydrated (Leica ST5020), and mounted (Leica MM24). Antibody specific positive control and negative control (omission of primary antibody) were parallel-stained.

\section{Scanning and analysis of IHC slides}

The IHC stained TMA slides were scanned into an Aperio slide scanner. The TMA are segmented and individual spots assigned to respective patients using a TMA lab software which works in conjunction with the spectrum software from Leica Aperio that analyzes the intensity of the staining of various proteins.

\section{Data analysis}

The raw $\mathrm{Cq}$ values from the miRNA assays were exported from the Bio-Rad CFX96 real-time PCR system (Carlsbad, CA) and data analyzed using the web-interface software available at http://pcrdataanalysis.sabiosciences. com/mirna following the manufacturer's instructions. Briefly, the data was normalized to the house keeping genes built within each array. Scatterplots of the miRNAs that were up- or down-regulated by 1.8 -fold compared to the control group along with the $p$-values were calculated using the web-interface software. The heatmap for miRNAs correlating the patient samples was generated using Matrix 2png, a utility for visualization of data matrix (http://www.chibi.ubc.ca/matrix2png/). All statistical analyses were carried out using GraphPad Prism (La Jolla, CA) by two-tailed student's $t$-test.

\section{ACKNOWLEDGEMENTS}

This research was supported by National Institutes of Health grants CA116984, CA123233 (to D.N.D), GM103639 (to D.N.D \& M.J) and Priority Research Centers Program (2009-0093820), the BK21 plus program (5256-20140100) through the National Research Foundation of Korea (to Y.S.S). We also thank the Stephenson Cancer Center, OUHSC, Oklahoma City, OK and an Institutional Development Award (IDeA) from the National Institute of General Medical Sciences of the National Institutes of Health under grant number P20 GM103639 for the use of Histology and Immunohistochemistry and Biospecimen Pathology Cores for all the services provided.

\section{CONFLICTS OF INTEREST}

The authors declare no conflict of interest.

\section{REFERENCES}

1. Siegel RL, Miller KD and Jemal A. Cancer Statistics, 2017. CA Cancer J Clin. 2017; 67(1):7-30.

2. Sonoda $Y$ and Barakat RR. Screening and the prevention of gynecologic cancer: endometrial cancer. Best Pract Res Clin Obstet Gynaecol. 2006; 20(2):363-377.

3. Ring KL, Garcia C, Thomas MH and Modesitt SC. Current and Future Role of Genetic Screening in Gynecologic Malignancies. Am J Obstet Gynecol. 2017; 9378(17):30512-4.

4. Tran AQ and Gehrig P. Recent Advances in Endometrial Cancer. F1000Res. 2017; 6:81.

5. Oliveto S, Mancino M, Manfrini N and Biffo S. Role of microRNAs in translation regulation and cancer. World $\mathrm{J}$ Biol Chem. 2017; 8(1):45-56. 
6. Yanokura M, Banno K, Iida M, Irie H, Umene K, Masuda $\mathrm{K}$, Kobayashi Y, Tominaga E and Aoki D. MicroRNAS in endometrial cancer: recent advances and potential clinical applications. EXCLI J. 2015; 14:190-198.

7. Fedor HL and De Marzo AM. Practical methods for tissue microarray construction. Methods Mol Med. 2005; 103:89101.

8. Mateescu B, Batista L, Cardon M, Gruosso T, de Feraudy Y, Mariani O, Nicolas A, Meyniel JP, Cottu P, Sastre-Garau $\mathrm{X}$ and Mechta-Grigoriou F. miR-141 and miR-200a act on ovarian tumorigenesis by controlling oxidative stress response. Nat Med. 2011; 17(12):1627-1635.

9. Liu TY, Zhang H, Du SM, Li J and Wen XH. Expression of microRNA-210 in tissue and serum of renal carcinoma patients and its effect on renal carcinoma cell proliferation, apoptosis, and invasion. Genet Mol Res. 2016; 15(1): 15017746 .

10. Song HM, Luo Y, Li DF, Wei CK, Hua KY, Song JL, Xu H, Maskey N and Fang L. MicroRNA-96 plays an oncogenic role by targeting FOXO1 and regulating AKT/FOXO1/Bim pathway in papillary thyroid carcinoma cells. Int J Clin Exp Pathol. 2015; 8(9):9889-9900.

11. Zheng Z, Ding M, Ni J, Song D, Huang J and Wang J. MiR142 acts as a tumor suppressor in osteosarcoma cell lines by targeting Rac1. Oncol Rep. 2015; 33(3):1291-1299.

12. Yan SY, Chen MM, Li GM, Wang YQ and Fan JG. MiR32 induces cell proliferation, migration, and invasion in hepatocellular carcinoma by targeting PTEN. Tumour Biol. 2015; 36(6):4747-4755.

13. Gao Y, Feng B, Han S, Zhang K, Chen J, Li C, Wang R and Chen L. The Roles of MicroRNA-141 in Human Cancers: From Diagnosis to Treatment. Cell Physiol Biochem. 2016; 38(2):427-448.

14. Li JZ, Li J, Wang HQ, Li X, Wen B and Wang YJ. MiR141-3p promotes prostate cancer cell proliferation through inhibiting kruppel-like factor-9 expression. Biochem Biophys Res Commun. 2017; 482(4):1381-1386.

15. Lin H, Dai T, Xiong H, Zhao X, Chen X, Yu C, Li J, Wang X and Song L. Unregulated miR-96 induces cell proliferation in human breast cancer by downregulating transcriptional factor FOXO3a. PLoS One. 2010; 5(12):e15797.

16. Wang Z, Yin B, Wang B, Ma Z, Liu W and Lv G. MicroRNA-210 promotes proliferation and invasion of peripheral nerve sheath tumor cells targeting EFNA3. Oncol Res. 2013; 21(3):145-154.

17. Puissegur MP, Mazure NM, Bertero T, Pradelli L, Grosso S, Robbe-Sermesant K, Maurin T, Lebrigand K, Cardinaud B, Hofman V, Fourre S, Magnone V, Ricci JE, et al. miR-210 is overexpressed in late stages of lung cancer and mediates mitochondrial alterations associated with modulation of HIF-1 activity. Cell Death Differ. 2011; 18(3):465-478.

18. Ma Z, Liu T, Huang W, Liu H, Zhang HM, Li Q, Chen $\mathrm{Z}$ and Guo AY. MicroRNA regulatory pathway analysis identifies miR-142-5p as a negative regulator of TGF- beta pathway via targeting SMAD3. Oncotarget. 2016; 7(44):71504-71513.

19. Saito Y, Suzuki H, Tsugawa H, Imaeda H, Matsuzaki J, Hirata K, Hosoe N, Nakamura M, Mukai M, Saito H and Hibi T. Overexpression of miR-142-5p and miR-155 in gastric mucosa-associated lymphoid tissue (MALT) lymphoma resistant to Helicobacter pylori eradication. PLoS One. 2012; 7(11):e47396.

20. Xia W, Zhou J, Luo H, Liu Y, Peng C, Zheng W and Ma W. MicroRNA-32 promotes cell proliferation, migration and suppresses apoptosis in breast cancer cells by targeting FBXW7. Cancer Cell Int. 2017; 17:14.

21. Jalava SE, Urbanucci A, Latonen L, Waltering KK, Sahu B, Janne OA, Seppala J, Lahdesmaki H, Tammela TL and Visakorpi T. Androgen-regulated miR-32 targets BTG2 and is overexpressed in castration-resistant prostate cancer. Oncogene. 2012; 31(41):4460-4471.

22. Gao J and Liu QG. The role of miR-26 in tumors and normal tissues (Review). Oncol Lett. 2011; 2(6):1019-1023.

23. Zuo K, Zhi K, Zhang X, Lu C, Wang S, Li M and He B. A dysregulated microRNA-26a/EphA2 axis impairs endothelial progenitor cell function via the p38 MAPK/ VEGF pathway. Cell Physiol Biochem. 2015; 35(2):477488.

24. Sakr M, Takino T, Sabit H, Nakada M, Li Z and Sato H. miR-150-5p and miR-133a suppress glioma cell proliferation and migration through targeting membranetype-1 matrix metalloproteinase. Gene. 2016; 587(2):155162.

25. Koshizuka K, Nohata N, Hanazawa T, Kikkawa N, Arai T, Okato A, Fukumoto I, Katada K, Okamoto Y and Seki N. Deep sequencing-based microRNA expression signatures in head and neck squamous cell carcinoma: dual strands of pre-miR-150 as antitumor miRNAs. Oncotarget. 2017; 8(18):30288-30304.

26. Wang X, Cao L, Wang Y, Wang X, Liu N and You Y. Regulation of let-7 and its target oncogenes (Review). Oncol Lett. 2012; 3(5):955-960.

27. Balzeau J, Menezes MR, Cao S and Hagan JP. The LIN28/ let-7 Pathway in Cancer. Front Genet. 2017; 8:31.

28. Luo M, Shen D, Wang W and Xian J. Aberrant expression of microRNA-26b and its prognostic potential in human cervical cancer. Int J Clin Exp Pathol. 2015; 8(5):55425548.

29. Majid S, Dar AA, Saini S, Deng G, Chang I, Greene K, Tanaka Y, Dahiya R and Yamamura S. MicroRNA-23b functions as a tumor suppressor by regulating Zeb1 in bladder cancer. PLoS One. 2013; 8(7):e67686.

30. Yan J, Jiang JY, Meng XN, Xiu YL and Zong ZH. MiR23b targets cyclin G1 and suppresses ovarian cancer tumorigenesis and progression. J Exp Clin Cancer Res. 2016; 35:31.

31. Goto Y, Kojima S, Nishikawa R, Enokida H, Chiyomaru T, Kinoshita T, Nakagawa M, Naya Y, Ichikawa T and 
Seki N. The microRNA-23b/27b/24-1 cluster is a disease progression marker and tumor suppressor in prostate cancer. Oncotarget. 2014; 5(17):7748-7759.

32. Du C, Lv Z, Cao L, Ding C, Gyabaah OA, Xie H, Zhou $\mathrm{L}, \mathrm{Wu} \mathrm{J}$ and Zheng S. MiR-126-3p suppresses tumor metastasis and angiogenesis of hepatocellular carcinoma by targeting LRP6 and PIK3R2. J Trans1 Med. 2014; 12:259.

33. Yagishita S, Fujita Y, Kitazono S, Ko R, Nakadate Y, Sawada T, Kitamura Y, Shimoyama T, Maeda Y, Takahashi F, Takahashi K, Tamura T and Koizumi F. ChemotherapyRegulated microRNA-125-HER2 Pathway as a Novel Therapeutic Target for Trastuzumab-Mediated Cellular Cytotoxicity in Small Cell Lung Cancer. Mol Cancer Ther. 2015; 14(6):1414-1423.

34. Zhang X, Tao T, Liu C, Guan H, Huang Y, Xu B and Chen M. Downregulation of miR-195 promotes prostate cancer progression by targeting HMGA1. Oncol Rep. 2016; 36(1):376-382.

35. Ding J, Huang S, Wang Y, Tian Q, Zha R, Shi H, Wang Q, Ge C, Chen T, Zhao Y, Liang L, Li J and He X. Genomewide screening reveals that miR-195 targets the TNF-alpha/ NF-kappaB pathway by down-regulating IkappaB kinase alpha and TAB3 in hepatocellular carcinoma. Hepatology. 2013; 58(2):654-666.

36. Wu K, Hu G, He X, Zhou P, Li J, He B and Sun W. MicroRNA-424-5p suppresses the expression of SOCS6 in pancreatic cancer. Pathol Oncol Res. 2013; 19(4):739-748.

37. Zhou Y, An Q, Guo RX, Qiao YH, Li LX, Zhang XY and Zhao XL. miR424-5p functions as an anti-oncogene in cervical cancer cell growth by targeting KDM5B via the Notch signaling pathway. Life Sci. 2017; 171:9-15.

38. Vosa U, Vooder T, Kolde R, Fischer K, Valk K, Tonisson N, Roosipuu R, Vilo J, Metspalu A and Annilo T. Identification of miR-374a as a prognostic marker for survival in patients with early-stage nonsmall cell lung cancer. Genes Chromosomes Cancer. 2011; 50(10):812-822.

39. Chen Y, Jiang J, Zhao M, Luo X, Liang Z, Zhen Y, Fu Q, Deng X, Lin X, Li L, Luo R, Liu Z and Fang W. microRNA-374a suppresses colon cancer progression by directly reducing CCND1 to inactivate the PI3K/AKT pathway. Oncotarget. 2016; 7(27):41306-41319.

40. Wu H, Liu Y, Shu XO and Cai Q. MiR-374a suppresses lung adenocarcinoma cell proliferation and invasion by targeting TGFA gene expression. Carcinogenesis. 2016; 37(6):567-575.

41. Qin X, Wan Y, Wang S and Xue M. MicroRNA-125a-5p modulates human cervical carcinoma proliferation and migration by targeting ABL2. Drug Des Devel Ther. 2016; 10:71-79.

42. Fan Z, Cui H, Xu X, Lin Z, Zhang X, Kang L, Han B, Meng J, Yan Z, Yan X and Jiao S. MiR-125a suppresses tumor growth, invasion and metastasis in cervical cancer by targeting STAT3. Oncotarget. 2015; 6(28):25266-25280.

43. Yoneyama K, Ishibashi O, Kawase R, Kurose $\mathrm{K}$ and
Takeshita T. miR-200a, miR-200b and miR-429 are onco-miRs that target the PTEN gene in endometrioid endometrial carcinoma. Anticancer Res. 2015; 35(3):14011410 .

44. Dalmay T. Mechanism of miRNA-mediated repression of mRNA translation. Essays Biochem. 2013; 54:29-38.

45. Wahid F, Shehzad A, Khan T and Kim YY. MicroRNAs: synthesis, mechanism, function, and recent clinical trials. Biochim Biophys Acta. 2010; 1803(11):1231-1243.

46. Brasseur K, Gevry N and Asselin E. Chemoresistance and targeted therapies in ovarian and endometrial cancers. Oncotarget. 2017; 8(3):4008-4042.

47. Kamat AA, Coffey D, Merritt WM, Nugent E, Urbauer D, Lin YG, Edwards C, Broaddus R, Coleman RL and Sood AK. EphA2 overexpression is associated with lack of hormone receptor expression and poor outcome in endometrial cancer. Cancer. 2009; 115(12):2684-2692.

48. Shang C, Lu YM and Meng LR. MicroRNA-125b downregulation mediates endometrial cancer invasion by targeting ERBB2. Med Sci Monit. 2012; 18(4):BR149-155.

49. Bamberger AM, Milde-Langosch K, Rossing E, Goemann $\mathrm{C}$ and Loning T. Expression pattern of the AP-1 family in endometrial cancer: correlations with cell cycle regulators. J Cancer Res Clin Oncol. 2001; 127(9):545-550.

50. Li C, Du X, Tai S, Zhong X, Wang Z, Hu Z, Zhang L, Kang P, Ji D, Jiang X, Zhou Q, Wan M, Jiang G and Cui Y. GPC1 regulated by miR-96-5p, rather than miR-182-5p, in inhibition of pancreatic carcinoma cell proliferation. Int $\mathrm{J}$ Mol Sci. 2014; 15(4):6314-6327.

51. Wang Y, Huang JW, Calses P, Kemp CJ and Taniguchi T. MiR-96 downregulates REV1 and RAD51 to promote cellular sensitivity to cisplatin and PARP inhibition. Cancer Res. 2012; 72(16):4037-4046.

52. Schwickert A, Weghake E, Bruggemann K, Engbers A, Brinkmann BF, Kemper B, Seggewiss J, Stock C, Ebnet K, Kiesel L, Riethmuller C and Gotte M. microRNA miR-142$3 p$ Inhibits Breast Cancer Cell Invasiveness by Synchronous Targeting of WASL, Integrin Alpha V, and Additional Cytoskeletal Elements. PLoS One. 2015; 10(12):e0143993.

53. Xiao P and Liu WL. MiR-142-3p functions as a potential tumor suppressor directly targeting HMGB1 in nonsmall-cell lung carcinoma. Int J Clin Exp Pathol. 2015; 8(9):10800-10807.

54. Lou K, Chen N, Li Z, Zhang B, Wang X, Chen Y, Xu H, Wang D and Wang H. MicroRNA-142-5p Overexpression Inhibits Cell Growth and Induces Apoptosis by Regulating FOXO in Hepatocellular Carcinoma Cells. Oncol Res. 2017; 25(1):65-73.

55. Shrestha A, Mukhametshina RT, Taghizadeh S, VasquezPacheco E, Cabrera-Fuentes H, Rizvanov A, Mari B, Carraro G and Bellusci S. MicroRNA-142 is a multifaceted regulator in organogenesis, homeostasis, and disease. Dev Dyn. 2017; 246(4):285-290.

56. Tsang FH, Au SL, Wei L, Fan DN, Lee JM, Wong CC, Ng 
IO and Wong CM. MicroRNA-142-3p and microRNA$142-5 p$ are downregulated in hepatocellular carcinoma and exhibit synergistic effects on cell motility. Front Med. 2015; 9(3):331-343.

57. Calin GA, Cimmino A, Fabbri M, Ferracin M, Wojcik SE, Shimizu M, Taccioli C, Zanesi N, Garzon R, Aqeilan RI, Alder H, Volinia S, Rassenti L, Liu X, Liu CG, Kipps TJ, et al. MiR-15a and miR-16-1 cluster functions in human leukemia. Proc Natl Acad Sci U S A. 2008; 105(13):51665171.

58. Pekarsky Y and Croce CM. Role of miR-15/16 in CLL. Cell Death Differ. 2015; 22(1):6-11.

59. Huang E, Liu R and Chu Y. miRNA-15a/16: as tumor suppressors and more. Future Oncol. 2015; 11(16):23512363.

60. Zhang Y, Liu Y and Xu X. Upregulation of miR-142$3 p$ Improves Drug Sensitivity of Acute Myelogenous Leukemia through Reducing P-Glycoprotein and Repressing Autophagy by Targeting HMGB1. Transl Oncol. 2017; 10(3):410-418.

61. Chen Y, Zhou X, Qiao J and Bao A. MiR-142-3p Overexpression Increases Chemo-Sensitivity of NSCLC by Inhibiting HMGB1-Mediated Autophagy. Cell Physiol Biochem. 2017; 41(4):1370-1382.

62. Bozok Cetintas V, Tetik Vardarli A, Duzgun Z, Tezcanli Kaymaz B, Acikgoz E, Aktug H, Kosova Can B, Gunduz $\mathrm{C}$ and Eroglu Z. miR-15a enhances the anticancer effects of cisplatin in the resistant non-small cell lung cancer cells. Tumour Biol. 2016; 37(2):1739-1751.

63. Huang N, Wu J, Qiu W, Lyu Q, He J, Xie W, Xu N and Zhang Y. MiR-15a and miR-16 induce autophagy and enhance chemosensitivity of Camptothecin. Cancer Biol Ther. 2015; 16(6):941-948. 